\title{
Saturated Subgraphs of the Hypercube
}

\author{
J. Robert Johnson and Trevor Pinto* \\ School of Mathematical Sciences, \\ Queen Mary University of London, \\ London E1 4NS, UK.
}

September 12, 2018

\begin{abstract}
We say a graph is $\left(Q_{n}, Q_{m}\right)$-saturated if it is a maximal $Q_{m}$-free subgraph of the $n$-dimensional hypercube $Q_{n}$. A graph is said to be $\left(Q_{n}, Q_{m}\right)$ semi-saturated if it is a subgraph of $Q_{n}$ and adding any edge forms a new copy of $Q_{m}$. The minimum number of edges a $\left(Q_{n}, Q_{m}\right)$-saturated graph (resp. $\left(Q_{n}, Q_{m}\right)$-semi-saturated graph) can have is denoted by $\operatorname{sat}\left(Q_{n}, Q_{m}\right)\left(\right.$ resp. $\left.s-\operatorname{sat}\left(Q_{n}, Q_{m}\right)\right)$. We prove that $\lim _{n \rightarrow \infty} \frac{\operatorname{sat}\left(Q_{n}, Q_{m}\right)}{e\left(Q_{n}\right)}=$ 0 , for fixed $m$, disproving a conjecture of Santolupo that, when $m=2$, this limit is $\frac{1}{4}$. Further, we show by a different method that $\operatorname{sat}\left(Q_{n}, Q_{2}\right)=$ $O\left(2^{n}\right)$, and that $s-s a t\left(Q_{n}, Q_{m}\right)=O\left(2^{n}\right)$, for fixed $m$. We also prove the lower bound $s-\operatorname{sat}\left(Q_{n}, Q_{m}\right) \geq \frac{m+1}{2} \cdot 2^{n}$, thus determining $\operatorname{sat}\left(Q_{n}, Q_{2}\right)$ to within a constant factor, and discuss some further questions.
\end{abstract}

2010 Mathematics Subject Classification: Primary 05C35, Secondary 05D05.

\section{Introduction}

Let $F$ be a (simple) graph. We say that a (simple) graph $G$ is $F$-free if it contains no subgraphs isomorphic to $F$. If $G$ is a maximal $F$-free subgraph of $H$, we say that $G$ is $(H, F)$-saturated. In other words, $G$ is $F$-saturated if it is a subgraph of $H$ and the addition of any edge from $E(H) \backslash E(G)$ forms a copy of $F$. In this context, $H$ is referred to as the host graph, $F$ as the forbidden graph and $G$ as a saturated graph.

The famous Turán problem in extremal combinatorics can be expressed naturally in the language of saturated graphs. The extremal number of $F, \operatorname{ex}\left(K_{n}, F\right)$, (often written as $e x(n, F)$ ) is usually defined as the maximum number of edges in an $F$-free subgraph of $K_{n}$. However, it can equivalently be written as:

$$
e x\left(K_{n}, F\right)=\max \left\{e(G): G \text { is }\left(K_{n}, F\right) \text {-saturated }\right\} .
$$

${ }^{*}$ Supported by an EPSRC doctoral studentship. 
This formulation yields a natural 'opposite' of the Turán problem. We define the saturation number of $F$, sat $(H, F)$ as:

$$
\operatorname{sat}(H, F)=\min \{e(G): G \text { is }(H, F) \text {-saturated }\} .
$$

A variant of this is the semi-saturation number, $s$-sat $(H, F)$. We say that a graph is $(H, F)$-semi-saturated if $G$ is a subgraph of $H$ and adding any edge from $E(H) \backslash E(G)$ increases the number of copies of $F$. A graph is $(H, F)$-saturated if and only if it is $(H, F)$-semi-saturated and $F$-free. We define:

$$
s \text {-sat }(H, F)=\min \{e(G): G \text { is }(H, F) \text {-semi-saturated }\} .
$$

The most frequently studied host graph is the complete graph, $K_{n}$. Since work in the area began with Erdôs, Hajnal and Moon [6], many others have studied s-sat $\left(K_{n}, F\right)$ and $\operatorname{sat}\left(K_{n}, F\right)$ : see for instance the survey articles of Pikhurko [10] and of J. Faudree, R. Faudree and Schmitt [7] and the references contained therein.

In the literature, $\operatorname{sat}\left(K_{n}, F\right)$ is often written as $\operatorname{sat}(n, F)$ and $\left(K_{n}, F\right)$ saturated is usually written as $F$-saturated. Since the results in this paper concern a different host graph, we will reserve this latter abbreviation for a different meaning.

A much studied variant of the Turán problem was initiated by Erdôs in [5] and expanded upon by Alon, Krech and Szabò [1]. For a fixed graph $F$, they ask for $\operatorname{ex}\left(Q_{n}, F\right)$, the maximum number of edges in an $F$-free subgraph of the $n$-dimensional hypercube, $Q_{n}$. The most natural case is $F=Q_{m}$, a fixed cube. This is wide open, even for the case $m=2$. The asymptotic edge density of a maximum $Q_{2}$-free graph, i.e. $\lim _{n \rightarrow \infty} \frac{e x\left(Q_{n}, Q_{2}\right)}{e\left(Q_{n}\right)}$ was conjectured by Erdôs [5] to be $\frac{1}{2}$. It is still unknown, despite the attention of many authors - see for instance the work of Balogh, Hu, Lidický and Liu [2] and of Brass, Harborth and Nienborg [3].

In this paper, we focus on the saturation and semi-saturation problems, where the host graph is the hypercube and the forbidden graph is a subcube. That is, we study $\operatorname{sat}\left(Q_{n}, F\right)$ and $s$-sat $\left(Q_{n}, F\right)$. For brevity, we shall often write $F$-saturated (resp. $F$-semi-saturated) rather than $\left(Q_{n}, F\right)$-saturated (resp. $\left(Q_{n}, F\right)$-semi-saturated) in the remainder of this paper, when the value of $n$ is clear or irrelevant.

The best result along these lines is that of Choi and Guan [4:

$$
\limsup _{n \rightarrow \infty} \frac{\operatorname{sat}\left(Q_{n}, Q_{2}\right)}{e\left(Q_{n}\right)} \leq \frac{1}{4} .
$$

A conjecture that this is best possible, due to Santolupo, was reported in [7. The same survey article posed the more general question of determining $\operatorname{sat}\left(Q_{n}, Q_{m}\right)$.

The main result of this paper, in Section 3, is the construction, for all fixed $m$, of $\left(Q_{n}, Q_{m}\right)$-saturated graphs of arbitrarily low edge density, thus both generalizing and improving the bound of Choi and Guan. 
Theorem 1. For fixed $m$,

$$
\lim _{n \rightarrow \infty} \frac{\operatorname{sat}\left(Q_{n}, Q_{m}\right)}{e\left(Q_{n}\right)}=0 .
$$

Slightly more precisely, we show $\operatorname{sat}\left(Q_{n}, Q_{m}\right) \leq \frac{c_{1}}{n^{c_{2}}} e\left(Q_{n}\right)$, where $c_{1}$ and $c_{2}$ are constants depending on $m$. In the case $m=2, c_{2}=6 / 7$; it is higher for larger values of $m$.

In Section 4 we prove a stronger bound for the semisaturation version of the problem.

Theorem 2. For all $n, m, s$-sat $\left(Q_{n}, Q_{m}\right)<\left(m^{2}+\frac{m}{2}\right) 2^{n}$.

In the same section, we adapt this proof in the $m=2$ case to remove all copies of $Q_{2}$ and thus prove a bound on $\operatorname{sat}\left(Q_{n}, Q_{2}\right)$ much stronger than that given by Theorem 1 .

Theorem 3. For all $n$, sat $\left(Q_{n}, Q_{2}\right)<10 \cdot 2^{n}$.

It is easy to see that both these theorems are best possible up to a constant factor, as all $\left(Q_{n}, Q_{m}\right)$-semi-saturated graphs have minimum degree $m-1$.

In Section 5, we will improve this trivial lower bound, by showing that

$$
\operatorname{s-sat}\left(Q_{n}, Q_{m}\right) \geq \frac{m+1}{2} 2^{n} .
$$

In Section 6, we discuss an extension to our zero density upper bound and raise some open questions.

We briefly mention here a somewhat related saturation problem on the cube. Here, $Q_{n}$ is considered as $\mathcal{P}(X)$, the power set of an $n$ element set, $X$. Let $F$ be a fixed poset. A family $\mathcal{A} \subseteq \mathcal{P}(X)$ is said to be $F$-saturated if there is no subfamily of $\mathcal{A}$ with the same poset structure as $F$, but adding any set to $\mathcal{A}$ destroys this property. Both the maximum and minimum size of such $\mathcal{A}$ have been studied - see for instance Katona and Tarján [8] for the former and Morrison, Noel and Scott [9] for the latter.

\section{Preliminaries}

In this section, we introduce terminology, notation and concepts that will be used frequently in the remainder of this paper.

The hypercube $Q_{n}$ is the graph with vertex set $\{0,1\}^{n}$, and with edges between each pair of vertices that differ in exactly one coordinate. Alternatively, the vertex set may be considered as $\mathbb{F}_{2}^{n}$, the $n$-dimensional vector space over the field with 2 elements. We write $e_{1}, \ldots, e_{n}$ for the canonical basis of $\mathbb{F}_{2}^{n}\left(e_{i}\right.$ is the vector with a 1 in the $i^{t h}$ coordinate, and 0's elsewhere). We can see that $x$ is adjacent to $y$ if and only if $y=x+e_{i}$, for some $i \in\{1, \ldots, n\}$.

A subcube of $Q_{n}$ is an induced subgraph isomorphic to $Q_{m}$, for some $m \leq n$. A set $S$ of vertices is the vertex set of a subcube if and only if there is some set 
of coordinates $J \subseteq[n]=\{1,2,3, \ldots, n\}$, and constants $a_{j} \in\{0,1\}$ for each $j \in J$ such that $\left(x_{1}, \ldots, x_{n}\right) \in S$ if and only if for all $j \in J, x_{j}=a_{j}$. Fixed coordinates are those coordinates in $J$, whereas free coordinates are coordinates that are not fixed. We can thus represent a subcube as an element of $\{0,1, *\}^{n}$, with stars in the free coordinates, and $a_{j}$ in the fixed coordinates. As edges can be thought of as $Q_{1}$ 's, we may represent edges as elements of $\{0,1, *\}^{n}$ in this way. We will say an edge or subcube lies along the directions $i_{1}, \ldots, i_{k}$ if these contain all the free coordinates of the edge or subcube. The weight of $x \in V\left(Q_{n}\right)$ is the number of coordinates of $x$ that are 1 .

We may write $Q_{n_{1}+n_{2}}$ as $Q_{n_{1}} \square Q_{n_{2}}$, the graph Cartesian product of $Q_{n_{1}}$ and $Q_{n_{2}}$. In other words, $Q_{n_{1}+n_{2}}$ is formed by replacing each vertex of $Q_{n_{2}}$ with a copy of $Q_{n_{1}}$. We call these principle $Q_{n_{1}}$ 's. Where there was a $Q_{n_{2}}$ edge $e$, we instead put edges between corresponding vertices of the principle $Q_{n_{1}}$ 's placed at the endpoints of $e$. So we have two types of edges: internal edges which have both endpoints in the same principle $Q_{n_{1}}$ and external edges which have endpoints in different principle $Q_{n_{1}}$ 's. Notice that there are $n_{1}$ directions along which internal edges lie, and $n_{2}$ directions along which external edges lie. This view of $Q_{n_{1}+n_{2}}$ is crucial in the proof of Theorem 1 1 we will write $Q_{n_{1}+n_{2}}$ as $Q_{n_{1}} \square Q_{n_{2}}$ when we wish to use this viewpoint.

Another way of encapsulating the product nature of $Q_{n}$ is to write a vertex $v$ as $\left(v_{1}\left|v_{2}\right| \ldots \mid v_{t}\right)$, where $v_{i} \in\{0,1\}^{n_{i}}=V\left(Q_{n_{i}}\right)$ and $n_{1}+\cdots+n_{t}=n$. Two vertices $\left(v_{1}\left|v_{2}\right| \ldots \mid v_{t}\right)$ and $\left(u_{1}\left|u_{2}\right| \ldots \mid u_{t}\right)$ are adjacent if and only if there is a $j$ such that $v_{j}$ and $u_{j}$ are adjacent as vertices of $Q_{n_{j}}$ and for all $i \neq j, v_{i}=u_{i}$. We will use this notation heavily in Section 4.

An object we shall use in several of our constructions is the Hamming code. The properties of Hamming codes that we require are listed below, but see van Lint 11 for more backgound. For our purposes, a Hamming code $C$ can be thought of as a subset of $V\left(Q_{n}\right)$, where $n=2^{r}-1$ for some $r$, with the following properties:

1. $C$ is a linear subspace of $\mathbb{F}_{2}^{n}$. More precisely, $C$ is the kernel of an $r$ by $n$ matrix $H$ over the field $\mathbb{F}_{2}$, called a parity check matrix. The columns of $H$ are precisely the non-zero vectors in $\mathbb{F}_{2}^{r}$.

2. $|C|=\frac{2^{n}}{n+1}$.

3. $C$ has minimum distance 3 . In other words, $\min \{d(x, y): x, y \in C\}=3$.

4. $C$ is a dominating set for $Q_{n}$. In other words, every vertex of $Q_{n}$ is either in $C$ or adjacent to a vertex in $C$.

Property 1 is usually taken as the definition of a Hamming code; the other properties are simple consequences of it.

A subset $C$ with these properties exists only if $n=2^{r}-1$ (and when it exists, it is the largest set with Property 3 , and the smallest with Property 4). For other values of $n$, we make do with an approximate Hamming code. This is any $C \subset V\left(Q_{n}\right)$ satisfying: 
1. $C$ is a linear subspace of $\mathbb{F}_{2}^{n}$. More precisely, $C$ is the kernel of an $r=$ $\lceil\log (n+1)\rceil$ by $n$ matrix $H$ over the field $\mathbb{F}_{2} . H$ has as columns any $n$ distinct binary vectors of length $r$.

2. $|C|=\frac{2^{n}}{2^{\left[\log _{2}(n+1)\right\rceil}}$.

3. $C$ has minimum distance 3 . In other words, $\min \{d(x, y): x, y \in C\}=3$.

\section{Zero density bound on $\operatorname{sat}\left(Q_{n}, Q_{m}\right)$}

In this section, we shall prove a quantitative version of Theorem 1, of which Theorem 1 is an immediate consequence.

Theorem 1. For all $m \geq 1$, there exist constants, $c_{m}$ and $a_{m}$, such that $\operatorname{sat}\left(Q_{n}, Q_{m}\right) \leq \frac{c_{m}}{n^{a} m} e\left(Q_{n}\right)$. More precisely, $a_{1}=1$ and $a_{m}=\frac{1}{7 \cdot 3^{m-2}}$, for all $m>1$.

Before discussing the proof of Theorem 1], we sketch a proof of the $\left(\frac{1}{4}+o(1)\right)$ bound of Choi and Guan, as this contains the main ideas of the proof of Theorem 1. This proof is significantly different from Choi and Guan's, which may be considered more direct. However, our approach, which uses $\frac{1}{3}+o(1)$ density saturated graphs to build $\frac{1}{4}+o(1)$ density saturated graphs, naturally gives rise to an iterative approach for proving Theorem 1 .

We assume that there exist three $\left(Q_{n}, Q_{2}\right)$-saturated graphs, $A_{1}, A_{2}$ and $A_{3}$ of $\frac{1}{3}+o(1)$ density, such that every edge of $Q_{n}$ lies in one of them. We will use these to produce a $\frac{1}{4}+o(1)$ density $\left(Q_{n+3}, Q_{2}\right)$-saturated graph $B^{\prime}$. These $A_{i}$ are relatively easy to construct - we will require a generalization of them in our proof of Theorem 1 .

We first construct an 'almost' $\left(Q_{n+3}, Q_{2}\right)$-saturated graph $B$. We consider $Q_{n+3}$ as $Q_{n} \square Q_{3}$. We leave two principle $Q_{n}$ 's corresponding to antipodal vertices of $Q_{3}$ empty. Around each of these empty $Q_{n}$, we arrange copies of $A_{1}, A_{2}, A_{3}$, as in the figure below. We also add all external edges with one endpoint in either of the two empty principle $Q_{n}$ 's (as indicated by the bold edges in the figure).

The graph constructed has the property that for any edge of an empty $Q_{n}$, $e$, the corresponding edge, $e^{\prime}$ is present in one of the $A_{i}$. So adding $e$ forms a $Q_{2}$ comprising $e, e^{\prime}$ and the two external edges that connect corresponding endpoints of $e$ and $e^{\prime}$. Since the $A_{i}$ are themselves $Q_{2}$-saturated graphs, adding any internal edge forms a copy of $Q_{2}$.

It is easy to see that $B$ is still $Q_{2}$-free, and a quick calculation shows that $B$ has edge density $\frac{1}{4}+o(1)$. We now prove a simple lemma that allows us to extend $B$ to a $Q_{2}$-saturated graph.

Lemma 4. Fix $m \geq 2$. Suppose that $G$ is a $Q_{m}$-free subgraph of $Q_{n}$ and $S \subseteq E\left(Q_{n}\right)$. Then we can form a $Q_{m}$-free graph $G^{\prime}$ by adding no more than $|S|$ edges to $G$ with the property that adding any edge in $S \backslash E(G)$ forms a copy of $Q_{m}$. 


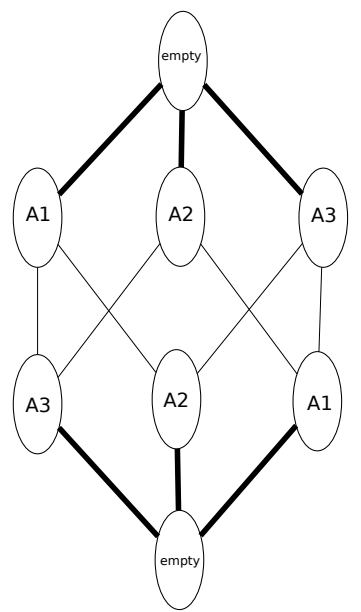

Figure 1: The 'almost' saturated graph, $B$

Proof. We order the edges in $S$ arbitrarily. Consider these edges in this order and add them to $G$ if and only if doing so does not form a copy of $Q_{m}$. Since only edges of $S$ are added by the process, we are done.

We apply this lemma to $B$, with $S$ being the set of external edges that have not already been added, i.e. those represented by the thin edges in Figure 1. This forms a $Q_{2}$-saturated graph, $B^{\prime}$. Since there are $\frac{3}{n+3} e\left(Q_{n+3}\right)$ external edges, the asymptotic edge density is still $\frac{1}{4}$.

The proof of Theorem 1 uses a similar method multiple times to produce $\left(Q_{n}, Q_{m}\right)$-saturated graphs of arbitrarily low density. In the case where $m=2$, we assume that we have a collection of $Q_{2}$-saturated graphs $A_{1}, \ldots, A_{k}$ of edge density at most $\rho$, such that every edge of $Q_{n}$ is contained in at least one of the $A_{i}$. We will view $Q_{n+k}$ as $Q_{n} \square Q_{k}$ and leave several principle $Q_{n}$ empty. We shall ensure that each empty $Q_{n}$ is adjacent, for every $i$, to a principle $Q_{n}$ filled with $A_{i}$, and add every external edge leaving these empty $Q_{n}$. This ensures that adding an edge within the empty $Q_{n}$ forms a copy of $Q_{2}$. The constraint on the empty principle $Q_{n}$ is that the set of vertices that we replace with empty $Q_{n}$ 's must have minimum distance 3 , and so we employ a Hamming code, enabling us to produce a graph with a lower density, $\rho^{\prime}$. Of course, to apply this method again, we need several $\left(Q_{n+k}, Q_{m}\right)$-saturated graphs of density $\rho^{\prime}$, which between them cover the edges of $Q_{n+k}$. This turns out to be not much harder, using cosets of the Hamming code.

In the general $m$ case we adapt this method. We would like to use a collection of $A_{i}$ that cover all the copies of $Q_{m-1}$ in $Q_{n}$. Such a collection seems hard to construct, but a modification of the argument shows that it suffices to cover almost all copies of $Q_{m-1}$. The other modification is that instead of using empty principle $Q_{n}$, we fill them with low density $Q_{m-1}$-saturated graphs, which we 
may assume exist by induction on $m$. We will use the following claim as a key part of the inductive step in proving the theorem.

Claim 1. Suppose we have a collection $A_{1}, \ldots, A_{k}$ of $\left(Q_{n}, Q_{m}\right)$-saturated graphs, each of density at most $\rho$, and some $n_{0}$ such that every $Q_{m-1}$ lies along the first $n_{0}$ directions is within one of these $A_{i}$. Suppose also that there is a $\left(Q_{n}, Q_{m}-1\right)$ saturated graph $G$ with no more than $\frac{c_{m-1}}{n^{a_{m-1}}} e\left(Q_{n}\right)$ edges. Then there is a collection of $k+1\left(Q_{n+k}, Q_{m}\right)$-saturated graphs, $B_{0}, \ldots, B_{k}$, such that every $Q_{m-1}$ that lies along the first $n_{0}$ directions is in one of these $B_{i}$. Further, each of the $B_{i}$ has density at most $\left(1-\frac{1}{2 k}\right) \rho+f\left(n, n_{0}\right)$, where $f$ is a function that tends to zero whenever $n, n_{0} \rightarrow \infty$ in such a way that $\frac{n_{0}}{n} \rightarrow 1$.

A precise upper bound on the densities of the $B_{i}$ is required for the quantitative part of the theorem; this will be stated at the end of the proof of this claim.

Proof of Claim 1. We start by constructing a $k+1$ colouring $c_{0}$ of $Q_{k}$, with the colours $0,1, \ldots, k$. Fix $C_{0}$, an approximate Hamming code in $Q_{k}$. We set $c_{0}(x)=0$ for all $x \in C_{0}$ and for all $j \in\{1, \ldots, k\}$ and all $x \in C_{0}$, we set $c_{0}\left(x+e_{j}\right)=j$. Note that when $k+1$ is not a power of 2 (i.e. when we do not have a genuine Hamming code), this colouring is not fully defined, since $C_{0}$ is not dominating. For now we assign arbitrary colours other than 0 to these vertices, but we will later decide on these colours.

We write $Q_{n+k}=Q_{n} \square Q_{k}$. We induce from $c_{0}$ a colouring on the set of principle $Q_{n}$ 's in the natural way. We start forming the graph $B_{0}$ by placing a copy of $A_{j}$ in each principle $Q_{n}$ coloured $j$, for each $j \neq 0$. Also, we add to the graph $B_{0}$ every external edge with one endpoint in a principle $Q_{n}$ coloured 0.

We place a graph isomorphic to $G$ in each $Q_{n}$ that is coloured 0 (we will choose which isomorphism later).

Notice that so far, $B_{0}$ is $Q_{m}$-free. Indeed, suppose that $B_{0}$ does contain a $Q_{m}$. This $Q_{m}$ cannot lie entirely within a single principle $Q_{n}$, by our assumption that the $A_{i}$ are saturated. As we have only added external edges that leave $Q_{n}$ coloured 0 , the $Q_{m}$ may contain an edge between two principle $Q_{n}$ 's only if one of them is coloured 0. Since the Hamming code has minimum distance 3, the $Q_{m}$ must contain edges in exactly two principle $Q_{n}$ 's, one of which is coloured 0 . But such $Q_{n}$ are $Q_{m-1}$-saturated and thus contain no $Q_{m-1}$, yielding a contradiction.

So far, $B_{0}$ is not quite $Q_{m}$-saturated - for instance adding an external edge may not create a copy of $Q_{m}$. However, we use Lemma 4 to remedy this. We add at most $\frac{k}{n+k} e\left(Q_{n+k}\right)$ edges to $B_{0}$ and we now only need to consider adding internal edges.

Adding an edge within a $Q_{n}$ coloured $j \neq 0$ forms a $Q_{m}$, as each $A_{j}$ is $Q_{m}$-saturated. Adding an edge within a principle $Q_{n}$ coloured 0 will form a $Q_{m-1}$ within that $Q_{n}$. If that $Q_{m-1}$ only uses edges in the first $n_{0}$ directions, it lies within one of the $A_{j}$ by the hypothesis of Claim 1. Since every principle $Q_{n}$ coloured zero is adjacent to a principle $Q_{n}$ of every non-zero colour, a $Q_{m}$ will be formed. Therefore, we only need to worry about adding edges to $G$ if 
the $Q_{m-1}$ formed does not lie exclusively along the first $n_{0}$ directions - we call such edges bad edges. We will now show that we may assume there are not very many bad edges.

Apply a random automorphism of $Q_{n}$ to $G$, our low density $Q_{m-1}$-saturated graph. We call the graph formed $G^{\prime} \subseteq Q_{n}$, which is to be placed within a principle $Q_{n}$ coloured 0 . Let $e$ be a fixed edge of this principle $Q_{n}$.

$$
\begin{aligned}
\mathbb{P}(e \text { is a bad edge }) & \leq 1-\frac{n_{0}}{n} \cdot \frac{n_{0}-1}{n-1} \cdots \cdots \frac{n_{0}-m+2}{n-m+2} \\
& \leq 1-\frac{\left(n_{0}-m\right)^{m-1}}{n^{m-1}} \\
& =\frac{n^{m-1}-\left(n_{0}-m\right)^{m-1}}{n^{m-1}} .
\end{aligned}
$$

This tells us that the expected number of bad edges, in each principle $Q_{n}$ coloured 0 , is no more than $\left(\frac{n^{m-1}-\left(n_{0}-m\right)^{m-1}}{n^{m-1}}\right) e\left(Q_{n}\right)$. We now choose the automorphism of $G$ that we left unspecified earlier; we can do this such that we get no more bad edges than the expected number. We use Lemma 4 , with $S$ being the set of bad edges, to form a graph that we also call $B_{0}$ that is $Q_{m}$-saturated.

We now construct the other $B_{i}$ to cover the required $Q_{m-1}$ 's. To construct $B_{i}$, we repeat the same method used for constructing $B_{0}$, except we use $C_{i}:=$ $\left\{c+e_{i}: c \in C_{0}\right\}$ instead of $C_{0}$. Note that we can make the arbitrary choices of colours to ensure each principle $Q_{n}$ is filled with each of the graphs $A_{1}, \ldots, A_{k}$, in one of the $B_{i}$.

It is easy to see that the $B_{i}$ satisfy the necessary $Q_{m-1}$ condition. Indeed any $Q_{m} \subseteq Q_{n+k}$ along the first $n_{0}$ directions must lie within a principle $Q_{n}$. When considered as a subgraph of this $Q_{n}$, it must lie in a copy of one of the $A_{i}$ - say $A_{j}$. This principle $Q_{n}$ is filled with $A_{j}$ in one of the $B_{i}$, so we are done.

It remains only to bound the number of edges in each saturated subgraph, $B_{i}$. Let $e(A)=\max \left\{e\left(A_{i}\right)\right\}, e(B)=\max \left\{e\left(B_{i}\right)\right\}, \rho(A)=\frac{e(A)}{n 2^{n-1}}$ and $\rho(B)=$ $\frac{e(B)}{(n+k) 2^{n+k-1}}$. In the calculations that follow, we write $a=a_{m-1}$ and $c=c_{m-1}$ for brevity.

Recall that edges were added to each $B_{j}$ in 4 ways: from copies of $A_{i}$, from adding external edges, from the $Q_{m-1}$-saturated graphs and from adding bad edges. 
Thus we have:

$$
\begin{aligned}
e(B) \leq & 2^{k}\left(1-\frac{1}{2^{\lceil\log (k+1)\rceil}}\right) e(A)+\frac{k}{n+k} e\left(Q_{n+k}\right) \\
& +\frac{2^{k}}{2^{\lceil\log (k+1)\rceil}} e\left(Q_{n}\right)\left(c_{m-1} n^{-a}+\frac{n^{m-1}-\left(n_{0}-m\right)^{m-1}}{n^{m-1}}\right) .
\end{aligned}
$$

Therefore,

$$
\begin{aligned}
\rho(B) \leq & \left(1-\frac{1}{2^{\lceil\log (k+1)\rceil}}\right) \rho(A)+\frac{k}{n+k} \\
& +\frac{1}{2^{\lceil\log (k+1)\rceil}}\left(c_{m-1} n^{-a}+\frac{n^{m-1}-\left(n_{0}-m\right)^{m-1}}{n^{m-1}}\right) \\
\leq & \left(1-\frac{1}{2 k}\right) \rho(A)+\frac{k}{n}+\frac{1}{k}\left(c_{m-1} n^{-a}+\frac{n^{m-1}-\left(n_{0}-m\right)^{m-1}}{n^{m-1}}\right) .
\end{aligned}
$$

Clearly if $n_{0}$ is large enough, and $n=(1+o(1)) n_{0}$, the last two terms can be arbitrarily small, thus concluding the proof of the claim.

We now return to prove Theorem 1 .

Proof of Theorem [1]. We use induction on $m$.

Base case: $m=1$. This is trivial - the subgraph of $Q_{n}$ with no edges is $Q_{1}$-saturated.

Inductive step: take $m>1$ and assume the Theorem holds for $m-1-$ i.e. there is a $\left(Q_{n}, Q_{m}-1\right)$-saturated graph $G$ with no more than $\frac{c_{m-1}}{n^{a_{m}-1}} e\left(Q_{n}\right)$ edges.

We first find a collection of subgraphs $A_{1}, \ldots, A_{m+1}$ of $Q_{n_{0}}$ that satisfy the hypothesis of Claim 1 with $\rho=1$. To do this, let $A_{i}$ initially consist of all edges whose lowest weight endpoint has weight in $\{i, \ldots, i+m-2\} \bmod m+1$, and then extend greedily until $A_{i}$ is $Q_{m}$ saturated. Each $A_{i}$ contains every $Q_{m-1}$ whose lowest weight vertex has weight $i \bmod m+1$, so every $Q_{m-1}$ is contained in one of these $A_{i}$. Trivially, we may bound the density of these $A_{i}$ above by 1 , and it is easy to see this is best possible up to a constant.

We now apply Claim 1 repeatedly, $t$ times. We write $k_{i}$ and $n_{i}$ for the value of $k$ and $n$ after the $i^{\text {th }}$ iterate. Clearly, $k_{i+1}=k_{i}+1, k_{0}=m+1, n_{i+1}=n_{i}+k_{i}$ and $n_{t}=n_{0}+\sum_{i=m}^{m+t} i=n_{0}+O\left(t^{2}\right)$.

After $t$ steps, we end with saturated graphs of density, $\rho$ : 


$$
\begin{aligned}
\rho & \leq \prod_{i=0}^{t-1}\left(1-\frac{1}{2 k_{i}}\right)+\sum_{i=0}^{t-1}\left(\frac{k_{i}}{n_{i}}+\frac{c_{m-1}}{k_{i}} \cdot n_{i}^{-a}+\frac{n_{i}^{m-1}-\left(n_{0}-m\right)^{m-1}}{k_{i} n_{i}^{m-1}}\right) \\
& \leq c \prod_{m=1}^{m+t}\left(1-\frac{1}{2 i}\right)+\frac{t(m+t+1)}{n_{0}}+\frac{t c_{m-1}}{m} \cdot n_{0}^{-a}+\frac{t}{m} \frac{n_{t}^{m-1}-\left(n_{0}-m\right)^{m-1}}{n_{0}^{m-1}} \\
& =c^{\prime} \cdot \exp \left(-\frac{1}{2} \sum_{i=1}^{t+m} \frac{1}{i}\right)+O\left(t^{2} n_{0}^{-1}\right)+O\left(t n_{0}^{-a}\right)+O\left(\frac{t^{3}}{n_{0}}\right) \\
& =c^{\prime \prime} t^{-\frac{1}{2}}+O\left(t n_{0}^{-a}\right)+O\left(t^{3} n_{0}^{-1}\right) .
\end{aligned}
$$

Here, $c, c^{\prime}$ and $c^{\prime \prime}$ are constants dependent on $m$. If $m=2$ it is optimal to take $t=n_{0}^{2 / 7}$, otherwise $a<\frac{3}{7}$, it is optimal to take $t=n_{0}^{2 a / 3}$.

This gives the required bound.

Note that the better bound for $\operatorname{sat}\left(Q_{n}, Q_{2}\right)$ in the next section can be fed into the induction in the theorem to produce the slightly better bound of $a_{m}=$ $\frac{1}{7 \cdot 3^{m-3}}$.

\section{Bounded average degree constructions}

\subsection{Semi-saturation}

In this section we will prove Theorem 2 , by constructing for each $m$ a family of $Q_{m}$-semi-saturated graphs with bounded average degree. Although it seems difficult in general to make these graphs $Q_{m}$-free, in the $m=2$ case we will use similar ideas to prove Theorem 3

In what follows it will be useful to write $n=m\left(2^{t}-1\right)+r$, where $0 \leq r<m 2^{t}$, and to let $n_{0}=2^{t}-1$. We write a vertex of $Q_{n}$ as $\left(v_{1}\left|v_{2}\right| \ldots\left|v_{m}\right| v_{m+1}\right)$, where $v_{i} \in\{0,1\}^{n_{0}}$ for $i \leq m$ and $v_{m+1} \in\{0,1\}^{r}$. The final section of the vector is only included to make the number of coordinates exactly $n$ but otherwise has no importance in the construction.

Proof of Theorem Q Let $C \subseteq\{0,1\}^{n_{0}}$ be a Hamming Code. We define:

$$
A=\left\{\left(v_{1}|\ldots| v_{m} \mid v_{m+1}\right) \in V\left(Q_{n}\right): \exists i \in\{1, m\} \text { such that } v_{i} \in C\right\} .
$$

We form $E(G)$ by picking all edges with at least one endpoint in $A$. Note that vertices in $A$ have degree $n$ in $G$; all other vertices have degree $m$. Therefore $e(G)=\frac{1}{2}\left((n-m)|A|+m 2^{n}\right) \leq \frac{m}{2}\left(n \frac{2^{n}}{\left(n_{0}+1\right)}+2^{n}\right)$. As $\frac{n}{n_{0}}<2 m, e(G)$ satisfies the bounds of the theorem.

We now show that $G$ is $Q_{m}$-semi-saturated. Assume $e \in E\left(Q_{n}\right) \backslash E(G)$ is along a direction $i$ in $\left\{1, n_{0}\right\}$ (all other cases can be dealt with similarly). We write the endpoints of the edges as $\left(v_{1}\left|v_{2}\right| \ldots v_{m} \mid v_{m+1}\right)$ and $\left(v_{1}^{\prime}\left|v_{2}\right| \ldots\left|v_{m}\right| v_{m+1}\right)$, 
where $v_{1}^{\prime}$ and all of the $v_{i}$ do not lie in $C$. Thus for $i=2,3, \ldots, m$ there exists $c_{i} \in C$ adjacent to $v_{i}$. Consider the $2^{m}$ points of the form $\left(x_{1}|\ldots| x_{m} \mid v_{m+1}\right)$, where $x_{1} \in\left\{v_{1}, v_{1}^{\prime}\right\}$ and for $i=2,3, \ldots, m, x_{i} \in\left\{v_{i}, c_{i}\right\}$. These vertices form a subcube of $Q_{n}$ and all but the endpoints of $e$ are in $A$. Thus when the edge $e$ is added, a copy of $Q_{m}$ is formed, concluding our proof.

Remark 5. Clearly, when $n=m\left(2^{t}-1\right)$ for some $t$, we get the slightly stronger bound s-sat $\left(Q_{n}, Q_{m}\right) \leq\left(\frac{m^{2}}{2}+\frac{m}{2}\right) 2^{n}$.

\subsection{Improved bound for $\operatorname{sat}\left(Q_{n}, Q_{2}\right)$}

In the $m=2$ case, the $Q_{2}$-semi-saturated graph constructed above consists of all edges incident with vertices in $A=\left\{\left(v_{1}\left|v_{2}\right| v_{3}\right) \in V\left(Q_{n}\right): v_{1} \in C\right.$ or $\left.v_{2} \in C\right\}$. It is easy to see this contains large subcubes, of the form $(c|*, \ldots, *| *, \ldots, *)$ or $(*, \ldots, *|c| *, \ldots, *)$, for $c \in C$. There are other $Q_{2}$ 's in this graph, but those within these large subcubes are hardest to deal with. We prevent subcubes of the first type by only adding edges of the form $\left\{(c \mid v),\left(c \mid v^{\prime}\right)\right\}$, where $c \in\{0,1\}^{n_{0}}$ and $v \in\{0,1\}^{n-n_{0}}$ and $v$ has lower weight than $v^{\prime}$, if $v_{1}$ has even weight. Of course doing just this alteration means the graph is no longer semi-saturated; we get around this by picking a subset $D$ of $V\left(Q_{n_{0}}\right)$ with similar properties to $C$, and adding edges starting at $\left(d\left|v_{2}\right| v_{3}\right)$ if $\left(v_{2} \mid v_{3}\right)$ contains an odd number of 1 's and if $d \in D$. We make use of the following claim, which allows us to choose a $D$ with the required properties.

Claim 2. There exists a $Q_{2}$-free spanning subgraph, $H$, of $Q_{n_{0}}$, that has two independent dominating sets, $C, D \subset V(H)=\{0,1\}^{n_{0}}$, with $C$ disjoint from $D$, where $|C|=2^{n_{0}} /\left(n_{0}+1\right)$ and $|D|=3 \cdot 2^{n_{0}} /\left(n_{0}+1\right)$. Further, $H$ only contains edges incident with $C \cup D$ and $e(H) \leq 2^{n_{0}+1}$.

We shall prove this claim later, but first we show why it implies the theorem.

Proof of Theorem 3. Similarly to before, we write $n=2\left(2^{t}-1\right)+r$, where $0 \leq r<2^{t+1}$, and let $n_{0}=2^{t}-1$. We write an element, $x$, of $\{0,1, *\}^{n}$ as $\left(x_{1}\left|x_{2}\right| x_{3}\right)$, where $x_{1}, x_{2} \in\{0,1, *\}^{n_{0}}$ and $x_{3} \in\{0,1, *\}^{r}$. We refer to $x_{1}$ as the first part of $x, x_{2}$ as the second part and so on. We will use this notation particularly when $x$ represents a vertex or an edge of $Q_{n}$ (it contains no stars or one star).

We start by constructing a graph $G$ that is $Q_{2}$-free and will then use Lemma 4 add a 'few' edges $\left(o\left(2^{n}\right)\right.$ edges) to form $G^{\prime}$, a $Q_{2}$-saturated graph. As in the proof of Theorem 2, we will define a subset, $A$ of the vertices, which will be dominating in $G$ :

$$
A=\left\{\left(v_{1}\left|v_{2}\right| v_{3}\right) \in\{0,1\}^{n}: v_{1} \in C \cup D \text { or } v_{2} \in C \cup D\right\} .
$$

The definition of $G$ is slightly more complicated. We add edges to $E(G)$ in three stages, and then delete some of these edges to ensure $G$ is $Q_{2}$-free. 
Firstly, we add all edges $e$ where $e_{1} \in C$, and the remainder, $\left(e_{2} \mid e_{3}\right)$, contains an even number of 1's and a single star, as well as edges where $e_{2} \in C$ and the remainder, $\left(e_{1} \mid e_{3}\right)$ contains an even number of 1's and a single star. We call these Type 1 edges. There are $2|C|\left(n-n_{0}\right) 2^{n-n_{0}-2} \leq \frac{\left(n-n_{0}\right)}{2\left(n_{0}+1\right)} 2^{n}$ Type 1 edges.

Similarly, we add those edges $e$ where $e_{1} \in D$ and the remainder, $\left(e_{2} \mid e_{3}\right)$ contains an odd number of 1's and a single star, as well as edges where $e_{2} \in D$ and the remainder contains an odd number of 1's and a single star. We call these Type 2 edges. There are $2\left(n-n_{0}\right)|D| 2^{n-n_{0}-2} \leq \frac{3\left(n-n_{0}\right)}{2\left(n_{0}+1\right)} 2^{n}$ Type 2 edges.

Lastly, we add all edges, $e$ where $e_{1}$ or $e_{2}$ is an edge of $H$. There are $2 \cdot 2^{n-n_{0}} e(H) \leq 4 \cdot 2^{n}$ Type 3 edges.

We now delete all edges $e$ which have an endpoint, $\left(v_{1}\left|v_{2}\right| v_{3}\right)$ such that both $v_{1}$ and $v_{2}$ lie in $C \cup D$. Thus $e(G) \leq\left(\frac{2\left(n-n_{0}\right)}{n_{0}+1}+4\right) 2^{n}-\frac{n 2^{n}}{\left(n_{0}+1\right)^{2}}$.

Suppose, for contradiction, that $G$ contains a $Q_{2}$. Note that as all edges of $G$ are incident with a vertex of $A$, this $Q_{2}$ must contain a vertex $\left(v_{1}\left|v_{2}\right| v_{3}\right) \in A$, where, without loss of generality, $v_{1} \in C \cup D$. Note that none of the vertices can have their second part in $C \cup D$, or there is a vertex of the $Q_{2}$ with both first and second part in $C \cup D$, impossible by our deletion step.

Let $s$ be the number of stars of the $Q_{2}$ that are in the first part of its vector representation. If $s=2$, all four edges are Type 3 edges, impossible as $H$ is $Q_{2}$-free.

If instead $s=1$, suppose the other star is in the second part (the other case is identical). Then we may write the vertices of the $Q_{2}$ as $\left(v_{1}\left|v_{2}\right| v_{3}\right),\left(v_{1}^{\prime}\left|v_{2}\right| v_{3}\right)$, $\left(v_{1}^{\prime}\left|v_{2}^{\prime}\right| v_{3}\right)$ and $\left(v_{1}\left|v_{2}^{\prime}\right| v_{3}\right)$, where $v_{1} \in C \cup D$ and $v_{2}, v_{2}^{\prime} \notin C \cup D$. It is easy to see that $v_{1}^{\prime} \in C \cup D$. By a parity argument, $v_{1}$ and $v_{1}^{\prime}$ are both in $C$ or both in $D$. But this is impossible as $C$ and $D$ are each $H_{0}$-independent sets.

Finally, if $s=0$, then we can have only Type 1 edges or only Type 2 edges (depending on whether $v_{1} \in C$ or $v_{1} \in D$ ). But this is impossible by a simple parity argument.

We now show that while $G$ is not quite saturated, it is 'almost' saturated. Suppose $e$ is a $Q_{n}$-edge not incident with $A$. Without loss of generality, the endpoints are $\left(v_{1}\left|v_{2}\right| v_{3}\right)$ and $\left(v_{1}^{\prime}\left|v_{2}\right| v_{3}\right)$, where $v_{1}, v_{1}^{\prime} v_{2}, v_{3} \notin C \cup D$. This is an element of $E\left(Q_{n}\right) \backslash E(G)$. Assume that $\left(v_{1} \mid v_{3}\right)$ is even, (the other case is very similar) and that $v_{1}^{\prime}$ has higher weight than $v_{1}$. Then pick $c \in C$ adjacent to $v_{2}$. $\left\{\left(v_{1}^{\prime}\left|v_{2}\right| v_{3}\right),\left(v_{1}^{\prime}|c| v_{3}\right)\right\}$ and $\left\{\left(v_{1}\left|v_{2}\right| v_{3}\right),\left(v_{1}|c| v_{3}\right)\right\}$ are Type 3 edges. Also, $\left\{\left(v_{1}|c| v_{3}\right),\left(v_{1}^{\prime}|c| v_{3}\right)\right\}$ is a Type 1 edge as $(x \mid y)$ is even. Thus a $Q_{2}$ would be formed by adding the edge.

All $Q_{n}$-edges with exactly one endpoint in $A$ are edges of $G$, so we only need to consider edges where one endpoint, $\left(v_{1}\left|v_{2}\right| v_{3}\right)$, has $v_{1}$ and $v_{2} \in C \cup D$. There are $\frac{2^{n}}{n}$ edges of this type, and so we may use Lemma 4 add them greedily to $G$ to form a $Q_{2}$-saturated graph $G^{\prime}$, which has no more edges than the bound in the theorem.

Remark 6. Again, we get a stronger bound for some values of $n$; when $n=$ $2\left(2^{t}-1\right)$ for some $t$, it is easy to see that $\operatorname{sat}\left(Q_{n}, Q_{2}\right) \leq 6 \cdot 2^{n}$. 
We now return to prove the claim.

Proof of Claim. Let $C$ be a Hamming code in $Q_{n_{0}}$. For $i=1, \ldots, n_{0}$, let $v_{i}$ be the image of the basis vector $e_{i}$ under the parity check matrix $M$ of the Hamming code. We may assume that $v_{1}=(1,0, \ldots, 0), v_{2}=(0,1,0, \ldots, 0)$ and $v_{3}=(1,1,0, \ldots, 0)$, as every vector in $\mathbb{F}_{2}^{t}$ occurs as a column of $M$. We shall construct $H$ in four stages, and then prove that it has the required properties.

1. Add to $E(H)$ every $Q_{n_{0}}$-edge adjacent to an element of $C$.

2. Add to $E(H)$ every $Q_{n_{0}}$-edge of the form $\left\{c+e_{1}+e_{k}, c+e_{1}\right\}$, where $c \in C$, and where $k \in\left[4, n_{0}\right]$ is such that $v_{k}$ has a 0 in the first coordinate.

3. Add to $E(H)$ every $Q_{n_{0}}$-edge of the form $\left\{c+e_{1}+e_{k}, c^{\prime}+e_{2}\right\}$, where $c, c^{\prime} \in C$, and where $k \in\left[4, n_{0}\right]$ is such that $v_{k}$ has a 1 in the first coordinate and a 0 in the second coordinate.

4. Add to $E(H)$ every $Q_{n_{0}}$-edge of the form $\left\{c+e_{1}+e_{k}, c^{\prime}+e_{3}\right\}$, where $c, c^{\prime} \in C$, and where $k \in\left[4, n_{0}\right]$ is such that $v_{k}$ has a 1 in the first coordinate and a 1 in the second coordinate.

Since $C$ is a Hamming code, it is an independent, dominating set and $|C|=2^{n_{0}} /\left(n_{0}+1\right)$. We write $C_{i}=\left\{c+e_{i}: c \in C\right\}$; in other words, $C_{i}=$ $M^{-1}\left(v_{i}\right)$. Let $D=C_{1} \cup C_{2} \cup C_{3}$. It is easy to see every edge of $H$ is incident with $C \cup D$. Since the $C_{i}$ are disjoint translates of $C$, a Hamming code, $|D|=3 \cdot 2^{n_{0}} /\left(n_{0}+1\right)$.

Again using that $C_{1}$ is a translate of a Hamming code, every $x \in V\left(Q_{n_{0}}\right) \backslash C_{1}$ can be written uniquely in the form $c+e_{1}+e_{k}$ for $c \in C$ and $k \in\left[1, n_{0}\right]$. The restriction $k \neq 1$ is equivalent to $x \notin C$. The restriction $k \neq 2$ is equivalent to $x \notin C_{3}$. This is as $M\left(c+e_{1}+e_{2}\right)=M(c)+M\left(e_{1}\right)+M\left(e_{2}\right)=v_{1}+v_{2}=v_{3}$. Similarly, $k=3$ if and only if $x \in C_{2}$. Thus steps 2,3 and 4 ensure $D$ is independent and dominating in $H$.

Notice also that each $x \notin C \cup D$ is $H$-adjacent to exactly 1 element in $D$. Hence $e(H) \leq 2\left|Q_{n_{0}}\right|$, as required. It remains only to show that $H$ is $Q_{2^{-}}$ free. Suppose not. Since we have only added edges with at least one endpoint in $C \cup D$, the $Q_{2}$ must contain two opposite vertices in $C \cup D$. Since $C$ has minimum distance 3 , and since every $x \notin C \cup D$ is adjacent to only 1 element in $D$, one of these vertices is in $D$, and one is in $C$. Thus the vertices of the $Q_{2}$ may be written in the form $c \in C, c+e_{i}, c+e_{j}$ and $c+e_{j}+e_{i} \in C_{k}$, where $i, j \in\left[4, n_{0}\right]$ are such that $v_{i}+v_{j}=v_{k}$, and $k \in\{1,2,3\}$. But it is impossible for all the edges of this $Q_{2}$ to lie in $e(H)$. Indeed, suppose for example that $k=3$. Then $v_{i}$ and $v_{j}$ must both have 1 in the first coordinate and 1 in the second coordinate, impossible if they sum to $v_{k}$. This concludes the proof of the claim. 


\section{Lower Bounds}

All the lower bounds in this section are for $s$-sat; easily $s$-sat $\left(Q_{n}, Q_{m}\right) \leq$ $\operatorname{sat}\left(Q_{n}, Q_{m}\right)$, so the bounds are also valid for sat.

If a graph is $\left(Q_{n}, Q_{m}\right)$-semi-saturated, for $m \geq 2$, it must be connected. Thus it contains a spanning tree for $Q_{n}$ and so $s$-sat $\left(Q_{n}, Q_{m}\right) \geq 2^{n}-1$. This shows that Theorems 2 and 3 are best possible up to a constant factor.

Another trivial observation improves this for $m \geq 3$ : if a graph is $\left(Q_{n}, Q_{m}\right)$ semi-saturated, it has minimum degree $m-1$. Thus $s$-sat $\left(Q_{n}, Q_{m}\right) \geq \frac{m-1}{2} 2^{n}$.

We do better than both trivial bounds for all $m$.

Theorem 7. If $m \geq 2$, s-sat $\left(Q_{n}, Q_{m}\right) \geq\left(\frac{m+1}{2}-o(1)\right) 2^{n}$.

Proof. Let $G$ be a $\left(Q_{n}, Q_{2}\right)$-semi-saturated graph with minimum degree $m-1$; note this contains all $\left(Q_{n}, Q_{m}\right)$-semi-saturated graphs. We call a pair $(v, e)$, where $\left.v \in V\left(Q_{n}\right), e \in E\left(Q_{n}\right) \backslash E(G)\right)$, good if there is a path of length 3 in $G$ linking the endpoints of $e$, that passes through $v$, meaning $v$ is not a start or end vertex of the path.

Note that every non-edge of $G$ is in at least 2 good pairs, whereas each vertex $v$ is in at most $\left(\begin{array}{c}d(v) \\ 2\end{array}\right)$ good pairs.

Therefore

$$
\sum_{v \in V\left(Q_{n}\right)}\left(\begin{array}{c}
d(v) \\
2
\end{array}\right) \geq 2\left(e\left(Q_{n}\right)-e(G)\right) .
$$

Subject to fixed $\sum_{v} d(v)$, the left hand side is maximized when the degrees are as different as possible. But no degree can be larger than $n$ or smaller than $m-1$. Note that $2 e(G)=\sum_{v} d(v)$, so we have $\frac{2 e(G)-2^{n}}{n-1}$ vertices of degree $n$ in this extreme case.

So certainly

$$
\begin{aligned}
\frac{2 e(G)-(m-1) 2^{n}}{n-1}\left(\begin{array}{l}
n \\
2
\end{array}\right) & \geq n 2^{n}-2 e(G) \\
(n+2) e(G)-n(m-1) 2^{n-1} & \geq n 2^{n} \\
e(G) & \geq\left(\frac{m+1}{2}-o(1)\right) 2^{n} .
\end{aligned}
$$

\section{$6 \quad$ Further Questions}

Having seen that $\lim _{n \rightarrow \infty} \frac{\operatorname{sat}\left(Q_{n}, Q_{m}\right)}{n 2^{n-1}}=0$, it is natural to ask for a more precise bound-while in Section 4 we have determined $\operatorname{sat}\left(Q_{n}, Q_{m}\right)$ up to a constant, for $m=2$, there is still a wide gap between the best upper and lower bounds for general $m$. In particular, we do not know whether families of $Q_{m}$-saturated graphs of bounded average degree exist for all $m$. 
Question 1. For which $m$ does there exist a constant $c_{m}$ such that for all $n$, $\operatorname{sat}\left(Q_{n}, Q_{m}\right) \leq c_{m} 2^{n}$ ?

In Section 4 , we were able to produce better bounds on $s$-sat $\left(Q_{n}, Q_{2}\right)$ than $\operatorname{sat}\left(Q_{n}, Q_{2}\right)$. Further, the construction we had for s-sat contained many copies of $Q_{2}$. This small amount of evidence may suggest that in general, the two are different, even asymptotically.

Question 2. Is sat $\left(Q_{n}, Q_{2}\right)=s$-sat $\left(Q_{n}, Q_{2}\right)$ for all $n$ ? Does equality hold for all sufficiently large $n$ ? If not, is $\lim \inf \frac{\operatorname{sat}\left(Q_{n}, Q_{2}\right)}{2^{n}}>\lim \sup \frac{s-\operatorname{sat}\left(Q_{n}, Q_{2}\right)}{2^{n}}$ ?

Recall that all our lower bounds are for $s$-sat-it seems hard to bound sat more strongly.

Another version of sat that has been studied in the literature (see Section 10 of [7]) (where the host graph is $K_{n}$ ) could be studied for this problem. We say that a graph $G \subseteq Q_{n}$ is $\left(Q_{n}, Q_{m}\right)$-weakly-saturated if we can add the edges in $E\left(Q_{n}\right) \backslash E(G)$ one at a time (in some order) such that every new edge creates at least one new copy of $F$. We write $w$-sat $\left(Q_{n}, Q_{m}\right)$ for the minimum number of edges a $\left(Q_{n}, Q_{m}\right)$-weakly saturated graph can have. Clearly, w-sat $\left(Q_{n}, Q_{m}\right) \leq$ $s$-sat $\left(Q_{n}, Q_{m}\right) \leq \operatorname{sat}\left(Q_{n}, Q_{m}\right)$. It is not hard to see, by induction on $n$, that there are many weakly $\left(Q_{n}, Q_{2}\right)$-saturated trees and so $w$-sat $\left(Q_{n}, Q_{2}\right)=2^{n}-$ 1. Indeed, given any $G_{1}, G_{2}$, possibly different weakly $\left(Q_{n-1}, Q_{2}\right)$-saturated trees, we place them in complementary $Q_{n-1}$ 's, and connect any one pair of corresponding vertices. This forms a weakly $\left(Q_{n}, Q_{2}\right)$-saturated tree. However, $w$-sat $\left(Q_{n}, Q_{m}\right)$ is in general not known.

Question 3. For $m \geq 3$, what is $w$-sat $\left(Q_{n}, Q_{m}\right)$ ?

In [1], Alon, Krech and Szabò discuss an interesting hypergraph type generalization of the Turán problem on the hypercube. We write $Q_{n}^{t}$ for the $2^{t}$-uniform hypergraph with vertex set $\{0,1\}^{n}$ and edge set consisting of all $t$-dimensional subcubes of $Q_{n}$. We say that a subhypergraph $H$ of $Q_{n}^{t}$ is $Q_{m}^{t}$-free if it contains no subhypergraph isomorphic to $Q_{m}^{t}$. As in the usual $(t=1)$ case of this Turán problem, they ask how many edges $H$ can have- in particular asking for the limit: $\lim _{n \rightarrow \infty} \max \left\{\frac{e(H)}{\left(\begin{array}{c}n \\ t\end{array}\right) 2^{n-t}}\right\}$. This question is still open, but it is interesting to know that the corresponding saturation problem can be attacked by the same method as the proof of Theorem 1 .

Let $H$ be a subhypergraph of $Q_{n}^{t}$. We say that $G$ is $\left(Q_{n}^{t}, Q_{m}^{t}\right)$-saturated if $G$ is $Q_{m}^{t}$-free but adding another $2^{t}$-edge to $G$ forms a subhypergraph isomorphic to $Q_{m}^{t}$. In other words, $G$ is a maximal $Q_{m}^{t}$-free subgraph of $Q_{n}^{t}$. We write $\operatorname{sat}\left(Q_{n}^{t}, Q_{m}^{t}\right)$ for the smallest number of edges a $\left(Q_{n}^{t}, Q_{m}^{t}\right)$-saturated $H$ can have. We can show by the same method as the proof of Theorem 1 that, for $t \geq 1$ and $s \geq 0$,

$$
\lim _{n \rightarrow \infty} \frac{\operatorname{sat}\left(Q_{n}^{t}, Q_{t+s}^{t}\right)}{\left(\begin{array}{l}
n \\
t
\end{array}\right) 2^{n-t}}=0
$$

As in the proof of Theorem 11 we proceed by induction on $s$ with the $s=0$ case being trivial. The iteration step analogous to Claim 1 is based on the same 
colouring of principal $Q_{n}$ 's. In each principal $Q_{n}$ with colour 0 we place a low density $Q_{t+s-1}^{t}$-saturated subgraph of $Q_{n}^{t}$. We also add all those $2^{t}$-edges which

contain $2^{t-1}$ points in some principal $Q_{n}$ with colour 0 . The remainder of the proof is a straightforward generalisation and the details are left to the reader.

\section{Acknowledgements}

The second author was supported by an EPSRC doctoral studentship.

\section{References}

[1] N. Alon, A. Krech, T. Szabò. Turán's theorem in the hypercube. SIAM J. Discrete Maths. 21(1) (2007) 66-72.

[2] J. Balogh, P. Hu, B. Lidický and H. Liu. Upper bounds on the size of 4and 6-cycle-free subgraphs of the hypercube. European J. Combin. 35 (2014) 75-85.

[3] P. Brass, H. Harborth and H. Nienborg. On the maximum number of edges in a $C_{4}$-free subgraph of $Q_{n} . \mathrm{J}$. Graph Theory. 19 (1) (1995) 17-23.

[4] S. Choi and P. Guan. Minimum Critical Squarefree Subgraph of a Hypercube. Proceedings of the Thirty-Ninth Southeastern International Conference on Combinatorics, Graph Theory and Computing. 189 (2008) 57-64.

[5] P. Erdôs. Some problems in graph theory, combinatorial analysis and combinatorial number theory. In Graph Theory and Combinatorics. B. Bollobás, ed. Academic Press, London. (1984) 1-17.

[6] P. Erdős, A. Hajnal and J. W. Moon. A Problem in Graph Theory. The American Mathematical Monthly. 71(10) (1964) 1107-1110.

[7] J. R. Faudree, R. J. Faudree and R. Schmitt. A Survey of Minimum Saturated Graphs. The Electronic Journal of Combinatorics. 18 (2011).

[8] G. O. H. Katona and T. G. Tarján. Extremal problems with excluded subgraphs in the $n$-cube. Graph Theory, Lagow, Poland, Lecture Notes in Mathematics. 1018, Berlin: Springer (1983), 84-93.

[9] N. Morrison, J. A. Noel and A. Scott. On Saturated k-Sperner Systems. arXiv:1402.5646. (2014).

[10] O. Pikhurko. Results and Open Problems on Minimum Saturated Hypergraphs. Ars Combinatorica. 72 (2004) 435-451.

[11] J. H. van Lint. Introduction to Coding Theory. Berlin; Springer-Verlag (1999). 